\title{
Efficacy of intraarterial chemoinfusion therapy for locally advanced breast cancer patients: a retrospective analysis of 28 cases
}

This article was published in the following Dove Press journal:

OncoTargets and Therapy

24 June 2013

Number of times this article has been viewed

\author{
Wei Zhangl,* \\ Rong Liu',* \\ Yingying Wang' \\ Sheng Qian' \\ Jianhua Wang' \\ Zhiping Yan' \\ Hongwei Zhang ${ }^{2}$ \\ 'Department of Interventional \\ Radiology, Zhongshan Hospital, \\ Fudan University, Shanghai, People's \\ Republic of China; ${ }^{2}$ Department of \\ General Surgery, Zhongshan Hospital, \\ Fudan University, Shanghai, People's \\ Republic of China \\ *These authors contributed equally to \\ this work
}

Correspondence: Jianhua Wang Department of Interventional Radiology, Zhongshan Hospital, Fudan University, No I 80 Fenglin Road,

Shanghai 200032,

People's Republic of China

Tel +8621 640419903088

Email wang.jianhua@zs-hospital.sh.cn
Objective: The objective of this study was to evaluate the outcome of image-guided delivery of intraarterially infused chemotherapeutic drugs for patients with locally advanced breast cancer.

Methods: Twenty-eight patients with pathologically proven, locally advanced breast cancer received intraarterial chemoinfusion therapy (chemoinfusion) with docetaxel $75 \mathrm{mg} / \mathrm{m}^{2}$ and epirubicin $50 \mathrm{mg} / \mathrm{m}^{2}$. Digital subtraction angiography was performed to determine tumor arterial blood supply and to guide chemotherapy infusion. Patients were evaluated for complete remission (CR) and partial remission (PR).

Results: Twenty-eight patients received a total of 64 intraarterial chemoinfusions, 20 patients (71.4\%) received two infusions, and eight patients $(28.6 \%)$ received three infusions. One patient (3.6\%) had CR and 23 (82.1\%) had PR. The total effectiveness rate (CR and PR) was $85.7 \%$ (24/28). All stage 3 patients underwent Phase II surgical resection after chemoinfusion, and the surgical resection participation rate was $100 \%(26 / 26)$. The mean time from the first chemoinfusion to surgery was $2 \pm 1.2$ months. Two patients with stage 4 cancer died of distant metastasis and cachexia, and the remaining 26 patients were still alive.

Conclusion: Intraarterial chemoinfusion is a safe and effective therapy, achieving down-staging in a relatively short period for locally advanced breast cancer.

Keywords: advanced breast cancer, intraarterial infusion, chemotherapy, therapeutic effect

\section{Introduction}

Breast cancer is one of the most common malignancies afflicting women worldwide, and its incidence has seen a steady increase over the years with the involvement of women at a much younger age, which poses a serious health threat to women and has a staggering global impact. ${ }^{1}$ Lymph node and hematogenous metastasis occurs at the early stage of breast cancer and is the principal cause of mortality of breast cancer patients., ${ }^{2,3}$ Surgical resection is the most effective method for the treatment of breast cancer, and adjuvant chemotherapy reduces the risk of disease recurrence and death in women who have operable breast cancer. ${ }^{4}$ For patients with inoperable breast cancer, adjuvant chemotherapy reduces tumor bulk and tumor stage, thereby increasing the chance of surgical resection and reducing postoperative tumor recurrence and metastasis. ${ }^{1-3}$ Local or regional chemotherapy is an alternative for reducing metastatic lesions and increasing patient survival. ${ }^{5}$ intra-arterial infusion chemotherapy (chemoinfusion), a form of regional chemotherapy, has been used in the treatment of gastrointestinal tumors, liver cancer, and pancreatic cancer. ${ }^{4}$ Transcatheter arterial chemoembolization play an important role in the treatment for surgically unresectable hepatocellular 
carcinoma. ${ }^{6}$ In the present retrospective study, we analyzed the outcome of image-guided delivery of intraarterially infused chemotherapeutic drugs for patients with locally advanced breast cancer.

\section{Patients and methods}

\section{Patients}

Twenty-eight patients with pathologically proven advanced breast cancer who were treated at the Department of Interventional Radiology, Zhongshan Hospital, between January 2007 and March 2011 were included in the retrospective analysis. The study protocol was approved by the local institutional review board at the authors' affiliated institution, and patient consent was not required because of the retrospective nature of the study. Patients were included if they (1) had stage 3 to 4 breast cancer (ipsilateral supraclavicular disease as the sole evidence of metastatic disease); (2) were between 16 and 75 years of age; (3) had received no prior chemotherapy, radiotherapy, or definitive surgical therapy for breast cancer; and (4) had adequate organ function. Tumors were staged according to the criteria of the Union for International Cancer Control (UICC). ${ }^{1-3}$ Tumor volume was determined by ultrasound or magnetic resonance imaging (MRI).

\section{Intraarterial chemoinfusion}

Twenty-seven patients were cannulated via the femoral artery and one patient via the radial artery ipsilateral to the lesion. Then, a 4F fixed-curve catheter (Cobra catheter, Cook Corporation, Bloomington, IN, USA) was advanced to the internal mammary artery ipsilateral to the lesion and to the subclavian artery. Digital subtraction angiography (DSA) was performed to determine tumor arterial blood supply. Chemoinfusion was decided by the major feeding artery. The intraarterial chemoinfusion regimen consisted of docetaxel $75 \mathrm{mg} / \mathrm{m}^{2}$ and epirubicin $50 \mathrm{mg} / \mathrm{m}^{2}$ in $200 \mathrm{~mL}$ of normal saline and 5\% glucose; the drugs were slowly infused via the catheter over at least 15 minutes. Intraarterial infusion was performed once every 3 weeks on average.

\section{Patient evaluation}

Patients were evaluated 2 to 3 weeks after the second intraarterial chemoinfusion. If the primary lesion and the involved lymph nodes shrank markedly in volume and skin and muscle involvement improved noticeably, patients were assigned to undergo surgical resection. Otherwise, patients were assigned to receive the third intraarterial chemoinfusion. Tumor volume and the size of lymph nodes were examined by ultrasound or MRI. Efficacy of treatment was assessed with the UICC criteria. Complete remission (CR) was defined as the complete disappearance of all clinical evidence of tumor and enlarged lymph nodes by clinical evaluation, mammogram, and ultrasound. Partial remission (PR) was defined as $\mathrm{a} \geq 50 \%$ decrease in the two longest perpendicular diameters in the tumor mass and local lymph nodes for a minimum of 4 weeks. Mild remission was defined as a $\leq 50 \%$ decrease in the two longest perpendicular diameters in the tumor mass and local lymph nodes for a minimum of 4 weeks. Stable disease was defined as no change in the two longest perpendicular diameters in the tumor mass and local lymph nodes for a minimum of 4 weeks. Progressive disease was defined as the appearance of any new lesions not previously identified or an increase in the two longest perpendicular diameters in the tumor mass and local lymph nodes for a minimum of 4 weeks.

\section{Statistical analysis}

Data were expressed as mean \pm standard deviation. The change in tumor size before and after intraarterial chemoinfusion was compared by use of a paired Student's $t$-test. The data were analyzed with SPSS version 16.0 (IBM Corporation, Armonk, NY, USA). A value of $P<0.05$ was considered statistically significant.

\section{Results}

\section{Patient demographic and baseline characteristics}

Twenty-eight patients were eligible for this retrospective analysis during the study period; all patients were female. Their mean age was 46 (range, 39-72) years. Twenty-seven patients had primary breast cancer, and one patient had recurrent breast cancer after breast-preserving surgery. The Eastern Cooperative Oncology Group score was 0 in 26 cases and 1 in two cases. The mean tumor size was $9.5 \pm 2.5 \mathrm{~cm}$ (range, 6-12 cm) in diameter. Eight patients $(36.4 \%, 8 / 22)$ had skin or chest muscle involvement with ulcer and apparent exudates. Twenty-two patients $(78.6 \%, 22 / 28)$ had ipsilateral or contralateral axillary lymph node enlargement. One patient $(3.6 \%, 1 / 28)$ had multiple metastases to the lungs, and one $(3.6 \%, 1 / 28)$ had metastasis to the liver. UICC stage 3 tumors were present in 26 patients $(92.3 \%, 26 / 28)$, including $3 \mathrm{~A}$ in two patients and $3 \mathrm{~B}$ in 24 patients, and stage 4 tumors were present in two patients $(7.1 \%, 2 / 28)$.

\section{DSA characteristics of the study subjects}

The primary lesions showed abundant blood supply, and all primary lesions were supplied by the branches of the 
internal mammary artery. Tumors in twelve patients $(42.9 \%$, $12 / 28$ ) were located in the upper outer quadrant and lower outer quadrant; metastases to the axillary, supraclavicular, and subclavian lymph nodes were observed in 22 patients $(78.6 \%, 22 / 28)$ and were supplied by the lateral thoracic artery or subscapular artery of the subclavian artery. DSA further revealed that arteries and their branches supplying tumors became thickened, varied in size, or were twisted and interrupted (Figures 1 and 2). The tumors had an irregular border and in the solid phase appeared as a patch or mass. Fistulae between the artery and vein were observed in four patients $(14.3 \%, 4 / 28)$ with advanced breast cancer. The axillary, supraclavicular, and subclavian lymph nodes with tumor metastases appeared isolated or fused.

\section{Outcomes of intraarterial chemoinfusion}

Chemotherapeutic drugs were allocated and infused according to DSA manifestations and tumor blood supply. Twenty-eight patients received a total of 64 intraarterial chemoinfusions, 20 patients $(71.4 \%, 20 / 28)$ received two infusions, and eight patients $(28.6 \%, 8 / 28)$ received

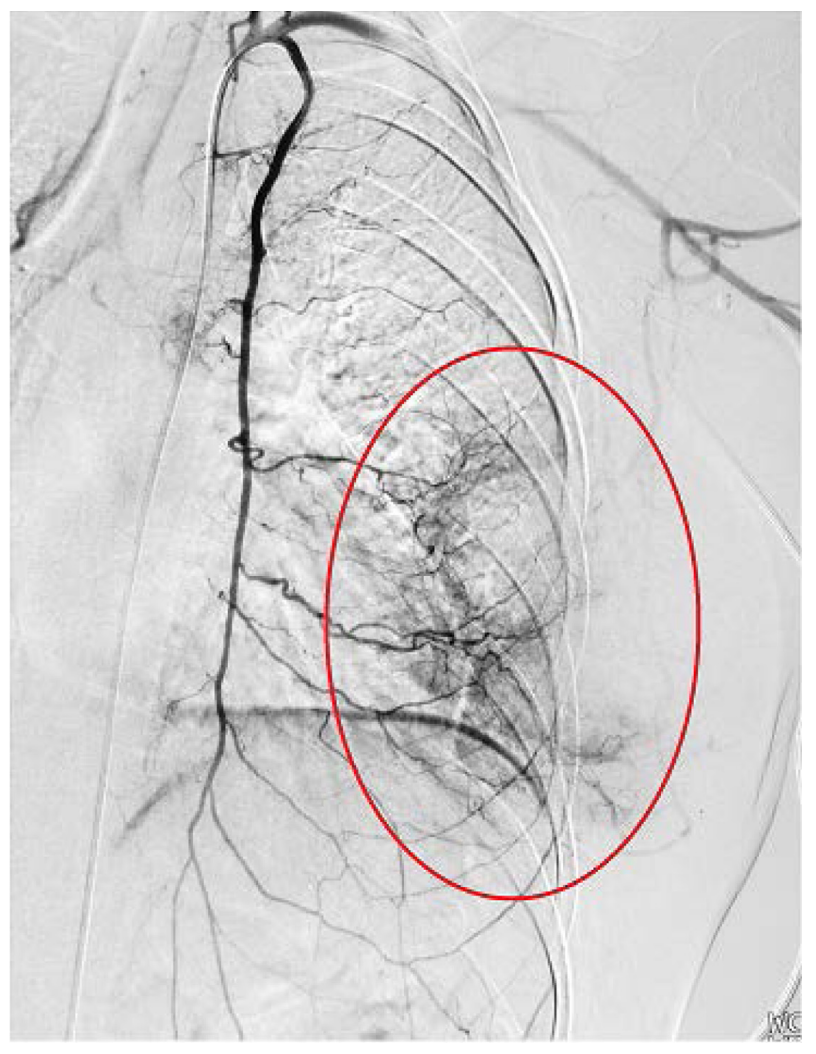

Figure I Digital subtraction angiography of the left internal mammary artery. Notes: Digital subtraction angiography of the left internal mammary artery reveals the extent of the tumor, with an approximate diameter of $9 \mathrm{~cm}$ supplied by multiple branches of the internal mammary artery. The tumor appears patchy, as indicated by the red oval.

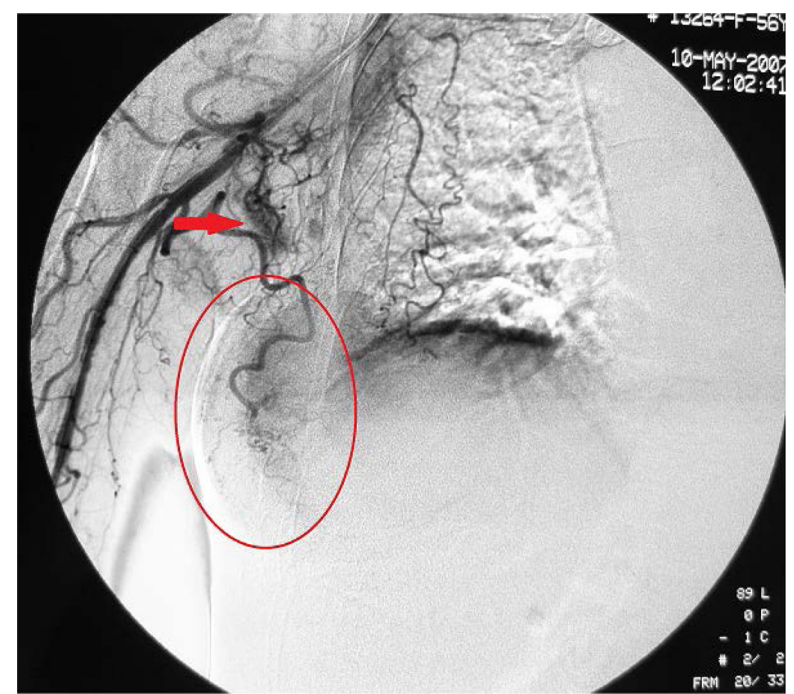

Figure 2 Digital subtraction angiography of the right subclavian artery.

Notes: Digital subtraction angiography reveals that the lateral thoracic artery, which supplies the tumor, becomes thickened and tortuous. The tumor appears patchy, with an approximate diameter of $7 \mathrm{~cm}$, as indicated by the red oval. Multiple lymph nodes appear nodular and are supplied by numerous smaller branches of the subclavian artery.

three infusions. Tumor volume after intraarterial chemoinfusion decreased to $3.4 \pm 1.6 \mathrm{~cm}$ (range, $0-5 \mathrm{~cm}$ ) in diameter, which was significantly smaller than before chemoinfusion $(P<0.05)$. Seven of eight $(87.5 \%)$ patients with skin and muscle invasion showed improvement in symptoms after chemoinfusion, and 16 of 22 patients (72.7\%) with axillary lymph node metastasis showed shrinkage in volume of lymph nodes. Local swelling and pain/discomfort improved or disappeared. Eighteen patients $(64.3 \%, 18 / 28)$ developed anorexia, vomiting, or fatigue, which resolved after 3 to 5 days of symptomatic treatment. Twelve patients (42.9\%, 12/28) showed local transient erythema. No patients developed skin ulcer or necrosis related to intraarterial chemoinfusion.

According to the UICC criteria, one patient (3.6\%, 1/28) had $\mathrm{CR}$ after intraarterial chemoinfusion, 23 (82.1\%, 23/28) had PR, two $(7.1 \%, 2 / 28)$ had mild remission, two $(7.1 \%$, $2 / 28$ ) had stable disease, and none had progressive disease. The total effective rate was $85.7 \%$ (24/28; Figures 3 and 4$)$. Twenty (76.9\%) of 26 stage 3 patients underwent surgery after two intraarterial chemoinfusions, and six (23.1\%) underwent surgery after three intraarterial chemoinfusions. The mean time from the first chemoinfusion to surgery was $2 \pm 1.2$ months. All stage 3 patients underwent Phase 2 surgical resection after chemoinfusion, and the surgical resection success rate was $100 \%(26 / 26)$. No patient developed serious complications; 22 patients received intravenous adjuvant chemotherapy, and two patients received 
A

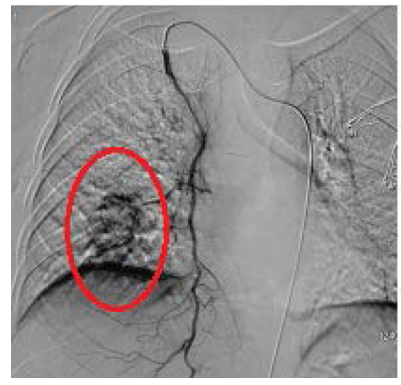

B

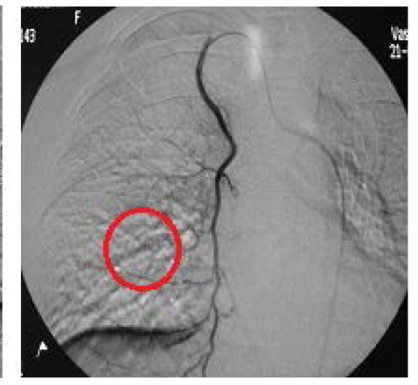

Figure 3 Digital subtraction angiography of the right internal mammary artery. Notes: (A) Digital subtraction angiography of the right internal mammary artery reveals a tumor mass with maximal diameter of $6 \mathrm{~cm}$ in a patient with stage 3A breast cancer before intraarterial chemoinfusion. The internal mammary artery sends out branches to supply the tumor, as indicated by the red oval. (B) The tumor mass disappeared after the second intraarterial chemoinfusion, as indicated by the red oval.

regional radiotherapy. At the time of writing this report, two stage 4 patients had died of distant metastasis and cachexia, and the remaining 26 patients were still alive.

\section{Discussion}

Locally advanced breast cancer is bulky in size, with a metastatic rate of $90 \%$ to regional lymph nodes, and it cannot be readily managed by Phase I surgical resection. ${ }^{7,8}$ Neoadjuvant chemotherapy is the standard treatment for locally advanced breast cancer; it reduces the size of the primary tumor lesion and the metastatic lymph nodes, down-stages breast cancer, and enhances the changes of Phase II surgical resection. Neoadjuvant chemotherapy also effectively reduces or abolishes micrometastases and suppresses tumor cell viability, thus lessening the probability of distant metastasis postoperatively. It also reveals chemosensitivity of a tumor, which facilitates postoperative adjuvant chemotherapy.
Similar to primary liver cancer, breast cancer has an abundant arterial blood supply that can be visualized by arterial DSA. Major feeding arteries of breast cancer tissue include internal branches of the internal mammary artery, the thoracic lateral artery, the intercostal artery, ${ }^{1-3,6}$ the subscapular artery, and the thoracoacromial artery. Breast cancer blood supply is closely related to tumor location: tumors in the outer quadrants are mainly supplied by the thoracic lateral artery and the subscapular artery, and tumors in the inner quadrants are mainly supplied by the internal thoracic artery and thoracoacromial artery. When the primary tumor is bulky in size, it can be fed by multiple arteries. In the present study, all 28 primary tumors were supplied by branches of the internal mammary artery, which indicates that the internal mammary artery is an important venue for intraarterial chemoinfusion. Twelve tumors (42.9\%) located in the upper outer quadrant and lower outer quadrant were supplied by the lateral thoracic artery or subscapular artery of the subclavian artery. Twenty-two axillary lymph nodes with metastases were supplied by branches of the subscapular artery.

Breast cancer has rich arterial supply, which lends itself to chemotherapy by intraarterial infusion. High concentrations of chemotherapeutic drugs reach the tumor interior via the feeding arteries, reaching a level two to ten times higher than that of adjacent tissues. Furthermore, direct contact of drugs with tumor cells more readily promotes apoptosis of tumor cells and inhibits tumor cell proliferation, thus lessening the metastasis and recurrence of the primary lesion. Intraarterial chemoinfusion for breast cancer can be traced back to the 1960s and is a form of regional chemotherapy that can readily achieve high local concentrations with fewer side effects. In the present study, 28 patients received a total

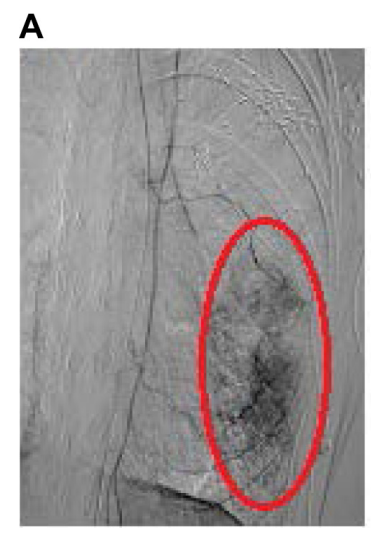

B

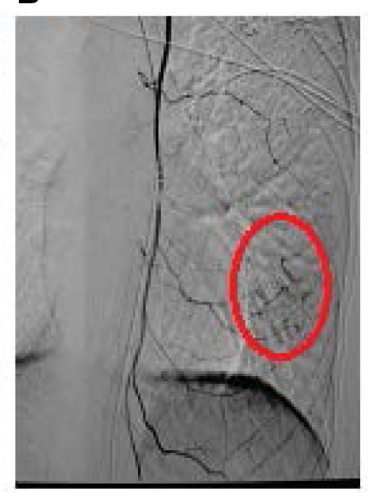

C

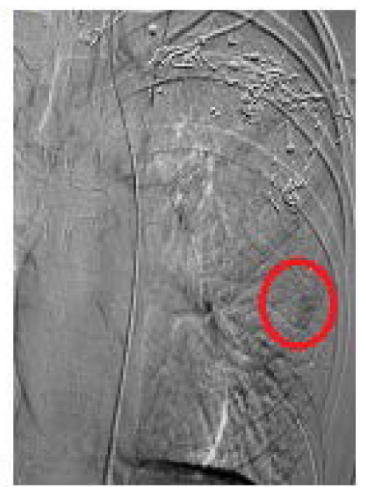

Figure 4 Digital subtraction angiography of the left internal mammary artery.

Notes: (A) Digital subtraction angiography of the left internal mammary artery reveals a tumor mass with an approximate diameter of II $\mathrm{cm}$ in a patient with stage $3 \mathrm{~B}$ breast cancer after breast-preserving surgery before intraarterial chemoinfusion. The tumor mass is adherent to the chest wall and is immobile, as indicated by the red oval. (B) The tumor mass has shrunk after the second intraarterial chemoinfusion, as indicated by the red oval. (C) The tumor mass has shrunk further after the third intraarterial chemoinfusion, as indicated by the red oval. 
of 64 intraarterial chemoinfusions with no development of serious complications such as skin ulcer or dermal necrosis. Twelve patients $(42.9 \%)$ showed local transient erythema, which disappeared after 1 or 2 days.

There have been several reports on regional intraarterial chemoinfusion for patients with advanced breast cancer. This approach reduces tumor volume, decreases the extent of tumor infiltration, and increases the rate of surgical resection. Kitagawa et $\mathrm{al}^{9}$ showed a marked reduction in tumor volume and a significant improvement in skin and muscle involvement, with a 3 -year overall survival rate of $71.4 \%$. We also demonstrated here that intraarterial chemoinfusion had a total effective rate of $85.7 \%$, and 26 stage 3 patients underwent surgical resection at a success rate of $100 \%$. The mean time from the first chemoinfusion to surgery was $2 \pm 1.2$ months. Our findings suggest that intraarterial chemoinfusion can shrink the tumor and metastatic lymph node volume in a short period of time and can down-stage tumors. In addition, intraarterial chemoinfusion could reduce the number of total cycles and doses of generalized intravenous chemotherapy needed for patients with locally advanced breast cancer with markedly fewer side effects and reduced time to surgery.

The present study is limited by the brief length of patient observation, which allows reporting only of the immediate outcomes of intraarterial chemoinfusion for locally advanced breast cancer. Furthermore, this is a single-arm study that was not compared against a group of patients receiving generalized intravenous chemotherapy.

In conclusion, intraarterial chemoinfusion is safe and effective for locally advanced breast cancer, reduces the bulk of primary tumor and metastasis to the lymph node in a short period of time, and reduces tumor staging, thus improving the resection rate and survival rate of cancer patients.

\section{Disclosure}

The authors report no conflicts of interest in this work.

\section{References}

1. Veronesi U, Boyle P, Goldhirsch A, Orecchia R, Viale G. Breast cancer. Lancet. 2005;365(9472):1727-1741.

2. Vinh-Hung V, Verschraegen C, Promish DI, et al. Ratios of involved nodes in early breast cancer. Breast Cancer Res. 2004;6(6): R680-R688.

3. Lee YT. Breast carcinoma pattern of metastasis at autopsy. J Surg Oncol. 1983;23(3):175-180.

4. Early Breast Cancer Trialists' Collaborative Group (EBCTCG). Effects of chemotherapy and hormonal therapy for early breast cancer on recurrence and 15-year survival: an overview of the randomised trials. Lancet. 2005;365(9472):1687-1717.

5. Buijs M, Kamel IR, Vossen JA, Georgiades CS, Hong K, Geschwind JF. Assessment of metastatic breast cancer response to chemoembolization with contrast agent enhanced and diffusion-weighted MR imaging. J Vasc Interv Radiol. 2007;18(8):957-963.

6. Ni S, Liu L, Shu Y. Sequential transcatheter arterial chemoembolization, three-dimensional conformal radiotherapy, and high-intensity focused ultrasound treatment for unresectable hepatocellular carcinoma patients. J Biomed Res. 2012;26(4):260-267.

7. Valero VV, Buzdar AU, Hortobagyi GN. Locally advanced breast cancer. Oncologist. 1996:1(1 and 2):8-17.

8. Singletary SE, Allred C, Ashley P, et al. Revision of American Joint Committee on Cancer staging system for breast cancer. J Clin Oncol. 2002;20(17):3628-3636.

9. Kitagawa K, Yamakado K, Nakatsuka A, et al. Preoperative transcatheter arterial infusion chemotherapy for locally advanced breast cancer (stage IIIb) for down-staging and increase of resectability. Eur J Radiol. 2002;43(1):31-36.
OncoTargets and Therapy

\section{Publish your work in this journal}

OncoTargets and Therapy is an international, peer-reviewed, open access journal focusing on the pathological basis of all cancers, potential targets for therapy and treatment protocols employed to improve the management of cancer patients. The journal also focuses on the impact of management programs and new therapeutic agents and protocols on

\section{Dovepress}

patient perspectives such as quality of life, adherence and satisfaction. The manuscript management system is completely online and includes a very quick and fair peer-review system, which is all easy to use. Visit http://www.dovepress.com/testimonials.php to read real quotes from published authors. 